\title{
Fuzzy based adaptive dimension parking algorithm including obstacle avoidance for autonomous vehicle parking
}

\author{
NAITIK M. NAKRANI \\ Electronics and Communication Dept. \\ Uka Tarsadia University \\ Bardoli, Gujarat \\ INDIA \\ MAULIN M. JOSHI \\ Electronics and Communication Dept. \\ Gujarat Technological University \\ SCET, Surat, Gujarat \\ INDIA
}

\begin{abstract}
Autonomous vehicle parking and obstacle avoidance navigation have drawn increased attention in recent times for autonomous vehicle-related solutions. Existing autonomous vehicle parking algorithms generally fail to mimic the human-like tendency to adapt naturally, and most of these designs are practically fixed. They do not preserve adaptive nature with machine dynamics, especially vehicles related. In this paper, a novel fuzzy-based adaptive dimension parking algorithm (FADPA) is proposed that integrates obstacle avoidance capabilities to a standalone parking controller that is made adaptive to vehicle dimensions in order to provide human-like intelligence for parking problems. This algorithm adopts fuzzy membership thresholds with respect to vehicle dimensions to enhance the vehicle's path during parking with taking care of obstacles. It is generalized for all segments of cars, and different simulation results are presented to show the effectiveness of the proposed algorithm.
\end{abstract}

Key-Words: - Autonomous vehicles, fuzzy systems, hybrid intelligent systems, adaptive systems, dynamic path planning, ultrasonic sensing.

Received: April 19, 2020. Revised: November 13, 2020. Accepted: December 1, 2020. Published: December 31, 2020.

\section{Introduction}

Autonomous vehicle parking system [1]-[25] should be capable of parking a vehicle without any human intervention at the same time should be capable of parking in any environmental conditions. These systems should address designs and implementation related heterogeneous issues like trajectory planning, steering control, and continuous scanning of the environment for dynamicity. Literature suggests that researchers have worked on them individually or in a combination of a few of them as multi-tasking. The major challenge to build these systems remains the dynamicity of the environment and the feedback control. Behaviorbased robot navigation architectures [11], [26]-[29] can be used as an integrated part vehicle parking system to track any unforeseen obstacle present in the surrounding. Navigation architectures required contactless sensing of the environment as an input, so many authors [10], [13], [23], [30]-[35] have used infrared sensors, ultrasonic sensors, LIDAR, laser sensors, and CCD sensor for their intelligent system design.

Literature suggests that numerous algorithms for parking problems involved fuzzy logic theory because of its ability to use linguistic information required for complex systems to formulate a controller's rule base. Human-like intelligence can be easily mimicked by machines via a fuzzy set theory. For obstacle avoidance based navigation, linguistic information obtained from sensors is fuzzified to select membership functions and values heuristically, by experimentation or expert rules. However, most of the time, these fuzzy membership values are fixed for a specific set of models.

An expert human driver can drive through optimal gaps with little sense of obstacles and understand vehicle dynamics. A drive-through experience is different whenever a person drives a 


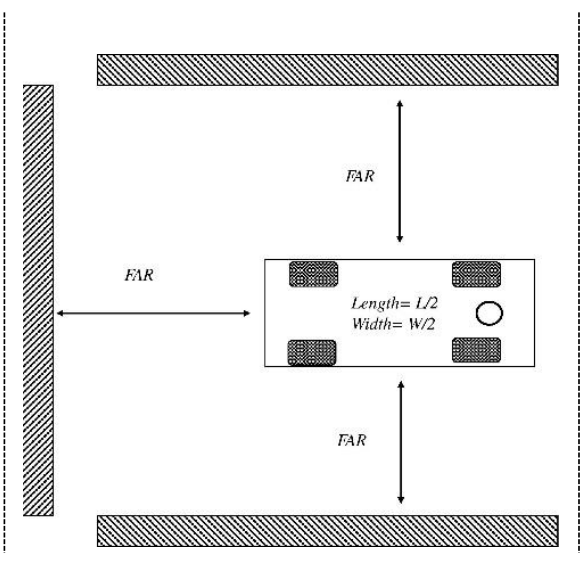

(a) Small size vehicle

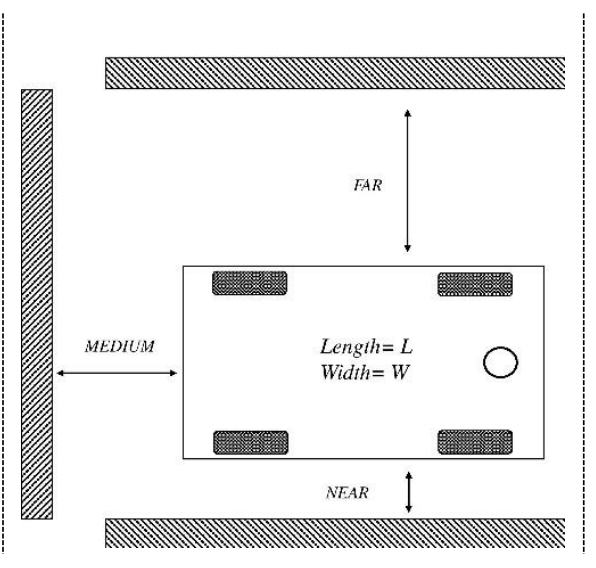

(b) Medium size vehicle

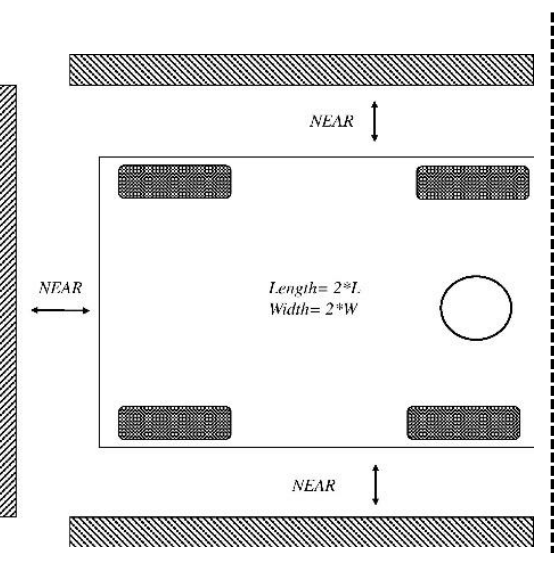

(c) Large size vehicle

Fig. 1. A typical "U shape" scenario indicating dynamic (different) inference of the same environment by different sized vehicles

small hatchback or large Special utility vehicles (SUVs). However, using such human-like intelligence by obstacle avoidance module of a parking model, one unique set of fuzzified membership ranges as incorporated in literature may not work for all different segments of vehicles, i.e., hatchback, sedan or SUV, etc. It should be noted that the perceptual inference of distances in the same fixed environment by different dimension vehicles is different, as conceptually shown in Fig. 1(a-c). These facts reveal that a predetermined and fixed set of thresholds used in existing models for fuzzy-based vehicle control must be made adaptive by considering parameters, including vehicle size.

In this paper, we propose a novel fuzzy-based adaptive dimension parking algorithm that utilizes a parameter derived from the vehicle's actual dimensions, provides the capability to adapt fuzzy variable membership values of parking module, and successfully parking a car with different sizes. In our earlier work [24], we developed a hybrid intelligent autonomous vehicle parking system with an inbuilt obstacle avoidance module. In this work, we integrate our proposed algorithm to the obstacle avoidance module. We have experimented with results with two different segments of the vehicle.

This paper's organization is as follows: In Section II, initially, a block diagram of the autonomous vehicle parking system is briefly discussed, and it is followed by an explanation of the proposed fuzzy-based adaptive dimension parking algorithm. In Section III, various scenarios for two different segments of vehicles are simulated in a confined environment with the presence of moving obstacles to visualize the application of the proposed algorithm. Finally, the conclusions are given in Section IV.

\section{Fuzzy based adaptive dimension parking algorithm (FADPA)}

Parking algorithms found in literature aim to solve the problem of fixed-sized vehicles. Once sensing of the surrounding environment is completed by sensors mounted on the vehicle, the next task is to infer them correctly to provide inputs to build models. However, threshold senor values to create such inference systems are heuristically or experimentally trained and are fixed in most cases. Due to these limitations, parking algorithms applicable for one set of fixed-size vehicles may not work efficiently if the vehicle dimensions are changed. The parking algorithm's perspective is altogether different tasks for a hatchback (small) car than a big sedan car while driving to narrow passes or to park along the curb or reverse. In this section, we propose an algorithm that can optimize its parameters according to the vehicle's size and will be able to park different dimension vehicles efficiently.

An intelligent autonomous parking system is developed, as shown in Fig. 2. An adaptive dimension parking controller executes fuzzy based adaptive dimension parking algorithm. This block diagram explains the working of a parallel parking controller to integrate an adaptive dimension parking controller. During parking maneuver, whenever the obstacles are sensed from the environment, a fuzzy controller called decision 
controller will switch Car-like mobile robot's (CLMR) command to the adaptive dimension parking controller. This controller's primary purpose is to detour CLMR safely away from the obstacles and avoid any collision with taking care of its final destination. Model of CLMR, sensors' calculations, detailed rule base, and working of these controller systems are explained in our earlier work [24]. The proposed work primarily focuses on the algorithm that is implemented to design an adaptive dimension controller, as shown in Fig. 2.

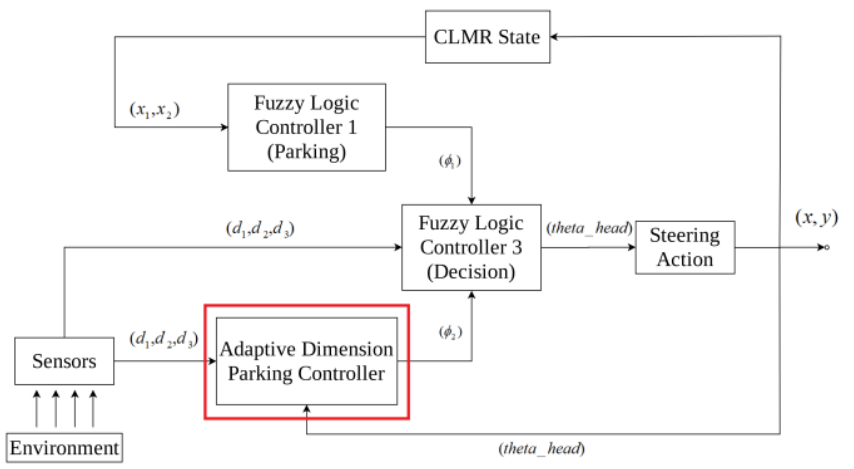

Fig. 2. Block diagram of a hybrid autonomous parking system

\subsection{Proposed Algorithm}

- Let car-like mobile robot (CLMR) be with the size of length $\mathrm{L}$ and width $\mathrm{W}$.

- Let total $\mathrm{S}_{\mathrm{N}}$ ultrasonic sensors be placed on the robot to sense the surrounding environment, as shown in Fig. 3. These sensors are represented as $\mathrm{S}_{1}, \mathrm{~S}_{2} \ldots \mathrm{S}_{\mathrm{k}}, \mathrm{S}_{\mathrm{k}+1} \ldots \mathrm{S}_{\mathrm{N}}$.

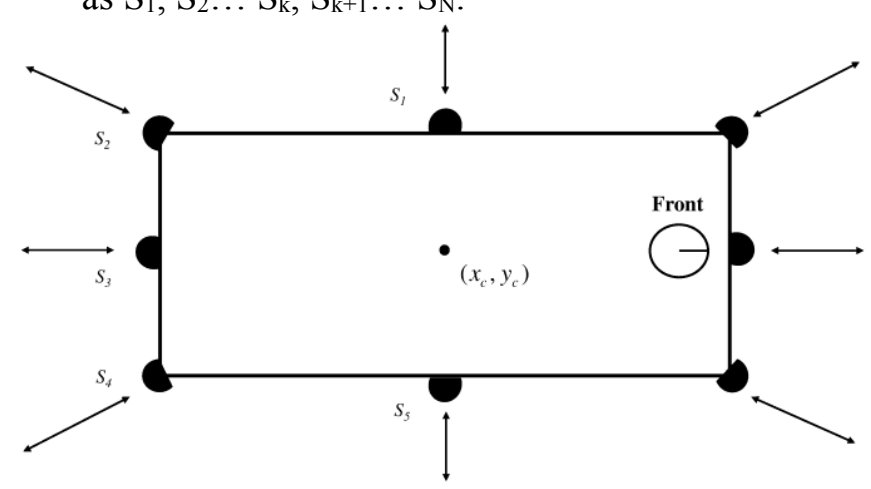

Fig. 3. Ultrasonic Sensor placement

- Let assume there are three different grouped sensor distances $d_{1}, d_{2}$, and $d_{3}$ obtained from $\mathrm{N}$ number of ultrasonic sensors mounted on peripheral of the vehicle as shown in Fig. 4

$$
\begin{aligned}
& d_{1}=\min \left\{d i s t_{-} S_{1}, d i s t_{-} S_{2}\right\} \\
& d_{2}=\min \left\{d i s t_{-} S_{2}, d i s t_{-} S_{3}, d i s t_{-} S_{4}\right\} \\
& d_{3}=\min \left\{d i s t_{-} S_{4}, d i s t_{-} S_{5}\right\}
\end{aligned}
$$
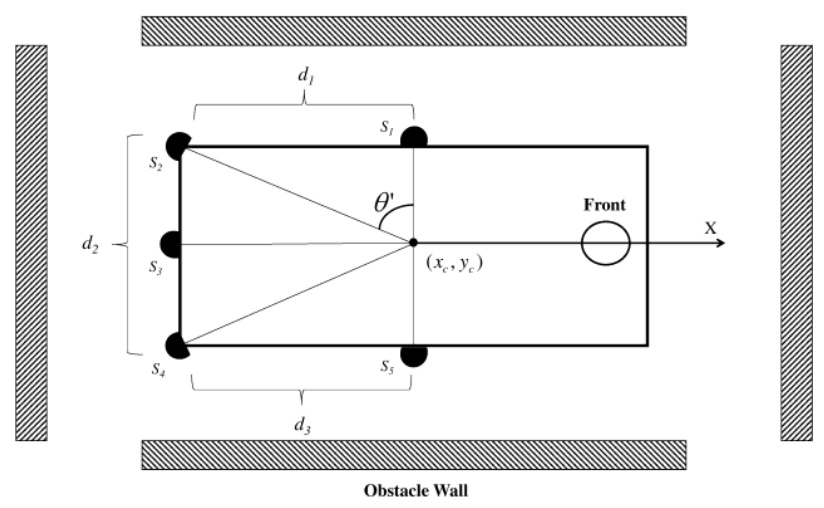

Fig. 4. Ultrasonic Sensors arrangement on CLMR

- All three distance groups are further fuzzified again into three different membership functions Near, Medium, and Far as defined below.

- Their thresholds are taken as a function of length $\mathrm{L}$ and width $\mathrm{W}$, and it is assumed to be constant for any vehicle size drive. These membership functions are defined as:

$$
\begin{aligned}
& \mu_{\text {near }}(x)=\operatorname{trap}\left(\frac{-L}{W}, 0, \frac{L}{W}-\frac{L}{4 W}, \frac{L}{W}\right) \\
& \mu_{\text {med }}(x)=\operatorname{trig}\left(\frac{L}{W}-\frac{L}{4 W}, \frac{L}{W}, \frac{L}{W}+\frac{L}{4 W}\right) \\
& \mu_{\text {far }}(x)=\operatorname{trap}\left(\frac{L}{W}, \frac{L}{W}+\frac{L}{4 W}, \frac{3 L}{W}, \frac{4 L}{W}\right)
\end{aligned}
$$

Where, $x \in\left[0, \frac{3 L}{W}\right]$ for all the distances

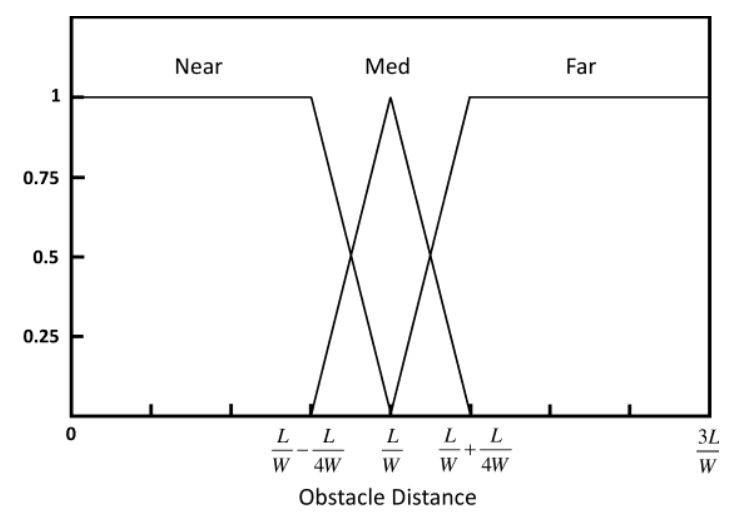

Fig. 5. Fuzzy Membership function for distances $\mathrm{d}_{1}, \mathrm{~d}_{2}$, and $\mathrm{d}_{3}$. 
The shape and value of membership functions used for distance $d_{1}, d_{2}$, and $d_{3}$ are shown in Fig. 5.

\subsection{Generalization Steps}

- We define a Constant $\mathrm{K}$, which is chosen from the actual vehicle dimension and used to modify thresholds of fuzzy membership functions varying according to vehicle dimension. Constant $\mathrm{K}$ can be taken from the range derived from given length (L) and width (W) as,

$$
\frac{W}{2 L} \leq K \leq \min \left(\frac{W}{L}, \frac{L-W}{L+W}\right)
$$

- Multiply, this factor " $K$ " with a threshold range of fuzzy membership functions as,

$$
\begin{aligned}
& \mu_{\text {near }}(x)=\operatorname{trap}\left(a^{\prime}, b^{\prime}, c^{\prime}, d^{\prime}\right) \\
& \quad \text { where, } \\
& a^{\prime}=K^{*} a ; b^{\prime}=K^{*} b ; c^{\prime}=K^{*} c ; d^{\prime}=K^{*} d \\
& \mu_{\text {med }}(x)=\operatorname{trig}\left(a^{\prime}, b^{\prime}, c^{\prime}\right) \\
& =\operatorname{trig}\left(K^{*} a, K^{*} b, K^{*} c\right) \\
& \mu_{\text {far }}(x)=\operatorname{trap}\left(a^{\prime}, b^{\prime}, c^{\prime}, d^{\prime}\right) \\
& =\operatorname{trap}\left(K^{*} a, K^{*} b, K^{*} c, K^{*} d\right) \\
& \text { Where, } x \in\left[0, \frac{3 L}{W} * K\right]
\end{aligned}
$$

- Execute parking of given CLMR, as shown in Fig. 2 with these modified threshold membership functions.

This algorithm is generalized for any dimension vehicle and can be used for any dynamic environment scenario.

\section{Simulation Results}

For demonstrating the proposed algorithm's practicality and efficacy, a parking scenario with a moving obstacle is created. Different cases are considered for parking of CLMR that exhibit the proposed algorithm's ability to execute additional capabilities like reverse parking, obstacle avoidance, redirection in case of non-feasibility of parking, etc. A Matlab simulation environment of size 22 by 22 $\mathrm{m}$ is created with a combination of a parking slot and static as well as moving obstacles. To match real vehicle dimension, CLMR length and width are taken as similar to Indian market available models Hyundai i20 (hatchback) and Hyundai Verna (sedan). The velocity of CLMR is taken as $1 \mathrm{~m} / \mathrm{s}$, and the vehicle is assumed to be equipped with ultrasonic sensors mounted on both front and rear sides to measure nearby obstacle distances. The velocity of a moving obstacle is considered lesser than the velocity of CLMR. The environment surface is assumed as flat.

Two different scenarios are considered to demonstrate the effect of applying the scaling factor ' $\mathrm{K}$ '. Both cases are simulated for different lengths and width of CLMR, different values of ' $K$ ' with moving obstacle. Results will show how effective and intelligently CLMR can pass through the narrow pass during parking, which is usually avoided with prefixed membership function thresholds. This algorithm is generalized and hence can be applied for commonly available different sized four-wheel autonomous vehicles.

\subsection{Case 1: Huyndai i20 Length-3996 mm, width- 1734 mm}

For this case, the L: $\mathrm{W}$ ratio is set to match with the real vehicle dimension of Hyundai i20 to fit in the environment setup shown in Fig. 6. Apart from static obstacles shown as boundaries of parking place, an additional moving obstacle is assumed to come in the path of CLMR being parked from the opposite end. CLMR is considered to be executing a reverse parking strategy for a given parallel parking algorithm. Further, for practical reasons moving obstacle's relative speed is assumed to be lesser than the rate of CLMR.

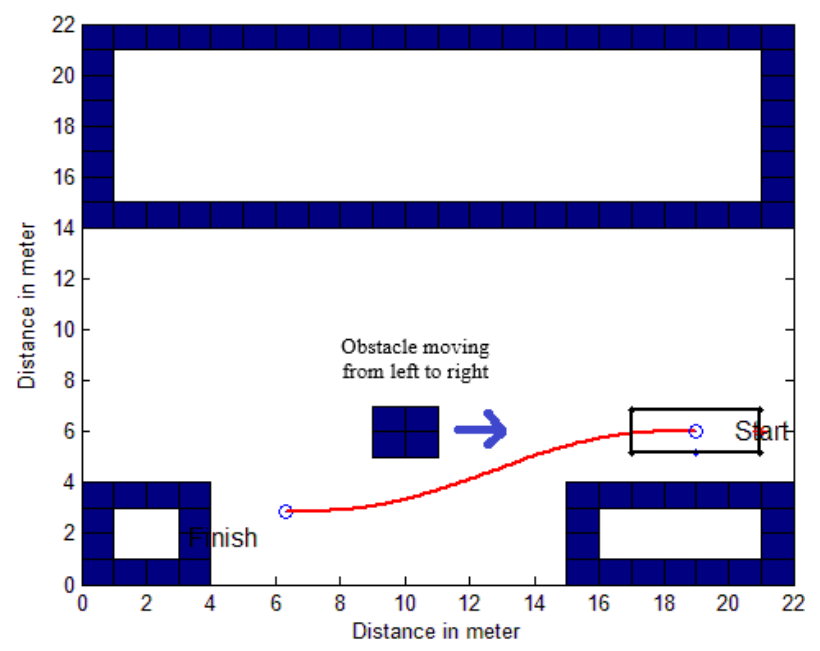

Fig. 6. A typical case scenario indicating initial and final locations of CLMR and initial position and direction of a moving obstacle. 
A typical case scenario indicating initial and final locations of CLMR and initial position and direction of a moving obstacle is shown in Fig. 6 . Fig. 7 and Fig. 8 highlight two different paths, followed by two CLMRs having different values of $\mathrm{K}$.

In the first case (having $\mathrm{K}$ equals 1), fuzzy variable membership thresholds are set as per (2), (3), and (4). Fig. 7(a-b) shows CLMR approaching parking (moving left). At the same time, moving obstacle (moving right) is continuously coming nearer to CLMR. Fig. 7(c) shows instant when a moving obstacle blocks the path of CLMR and finding no direct path CLMR starts moving right (start of obstacle avoidance behavior ). Fig. 7(d-f) show successful obstacle avoidance of CLMR. After resuming reverse parking, as shown in Fig. $7(\mathrm{~g}-\mathrm{h})$, finally, CLMR reaches the desired parking location as per Fig. 7(i).

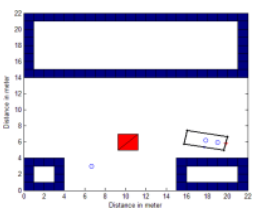

(a)

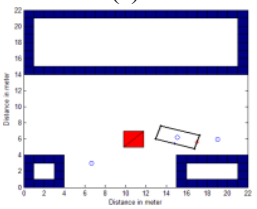

(d)

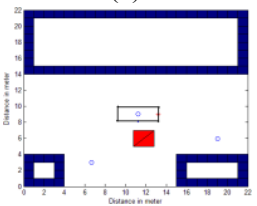

(g)

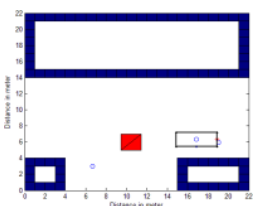

(b)

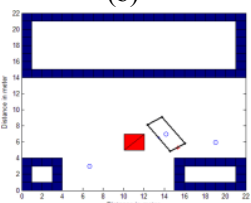

(e)

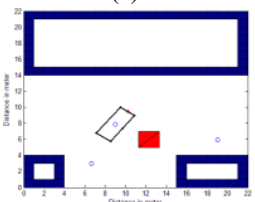

(h)

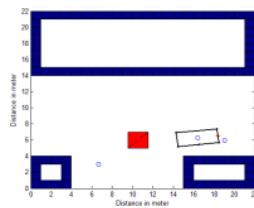

(c)

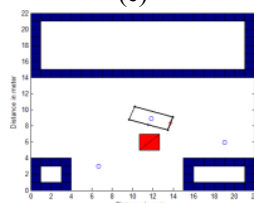

(f)

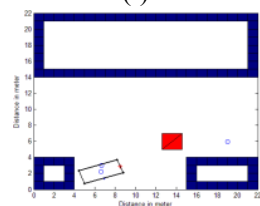

(i)
Fig. 7. Step sequence of reverse parallel parking with $\mathrm{K}=1$, no scaling

In the second case ( $\mathrm{K}$ is selected as 0.25 ), fuzzy variable membership thresholds are set as per (6), (7), and (8). With these values, the inference is changed, and CLMR tries to detour the optimal path while parking. In this case, also, Fig. 8(a-b) shows CLMR approaching parking (moving left). At the same time, moving obstacle (moving right) is continuously coming nearer to CLMR. Here, Fig. 8(c) shows instant when moving obstacle tries to block the path, but still, CLMR is finding sufficient space to maneuver in between moving obstacle and wall (target steering behavior). Fig. 8(d-f) shows successful maneuvering of CLMR still under the moving obstacle's influence. In Fig. 8(g-h) CLMR continues reverse parking, and finally, CLMR reaches the desired parking location as per Fig. 8(i).

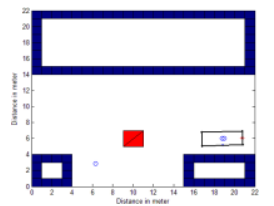

(a)

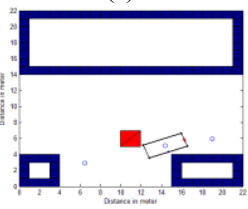

(d)

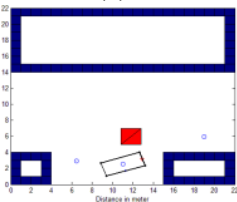

(g)

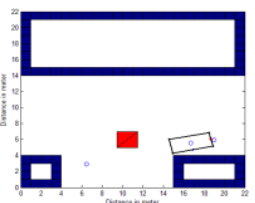

(b)

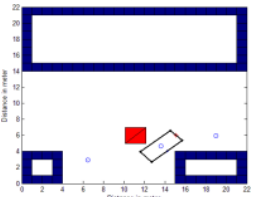

(e)

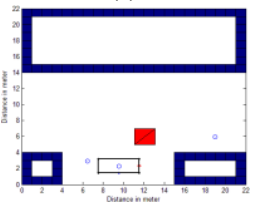

(h)

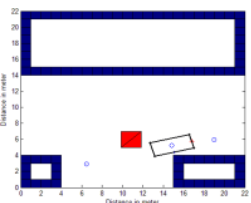

(c)

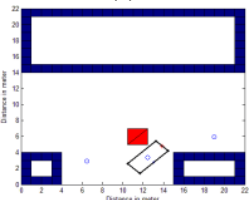

(f)

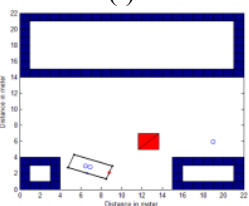

(i)
Fig. 8. Step sequence of reverse parallel parking with $\mathrm{K}=0.25$

\subsection{Case 2: Hyundai Verna Length-4440 mm, width-1729 mm}

For this case, L: W ratio is set to match Hyundai Verna's real vehicle dimension to fit in the environment setup shown in Fig. 9. To check its outcome with hatchback size vehicle, all the environment condition taken for this case is similar to Hyundai i20 scenario. A typical case scenario indicating initial and final locations of CLMR and initial position and direction of a moving obstacle is shown in Fig. 9. Fig. 10 and Fig. 11 highlight two different paths followed by two CLMRs having different $\mathrm{K}$ values.

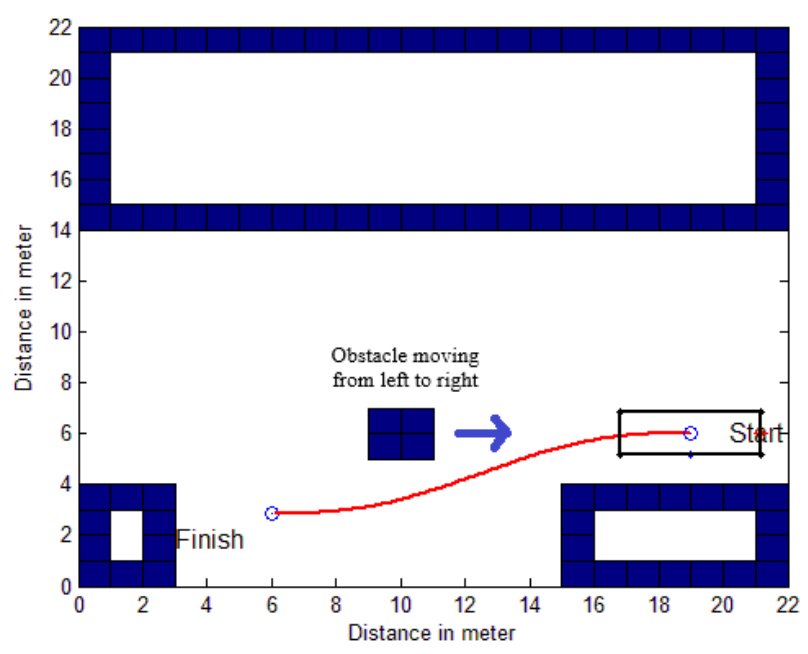

Fig. 9. A typical case scenario indicating initial and final locations of CLMR and initial position and 
direction of a moving obstacle.

In the first case (having $\mathrm{K}$ equals 1), fuzzy variable membership thresholds are set as per (2), (3), and (4). As the dimension of Hyundai Verna is higher than that of the Hyundai i20, the inference of fuzzy thresholds are high. Due to the result, CLMR takes a path to avoid moving obstacle with higher safety of margin. Step by step sequence of CLMR steering action is shown in Fig. 10(a-i).

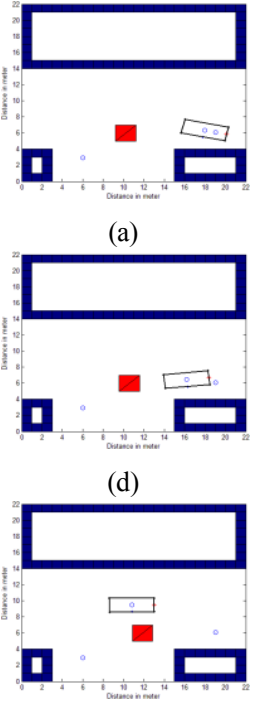

(g)

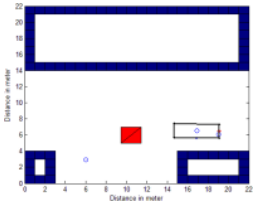

(b)

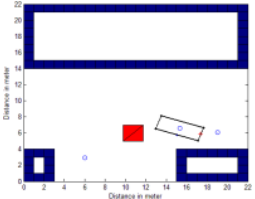

(e)

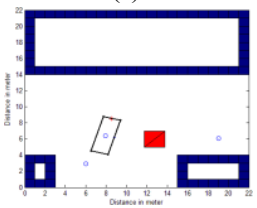

(h)

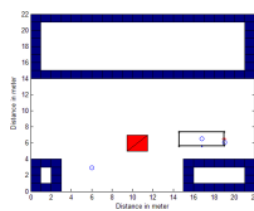

(c)

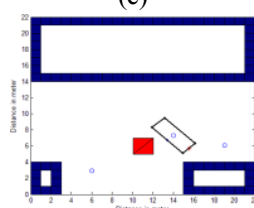

(f)

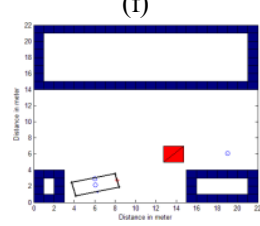

(i)
Fig. 10. Step sequence of reverse parallel parking with $\mathrm{K}=1$, no scaling

In the second case ( $\mathrm{K}$ is selected as 0.25 ), fuzzy variable membership thresholds are set as per (6), (7), and (8). With these values, the inference is changed and CLMR tries to detour the optimal path while parking. Even though fuzzy thresholds are higher compared to hatchback segment vehicles, Fig. 11(a-i) show CLMR approaching towards parking (moving left) with the optimal route as space is available to move between obstacles.

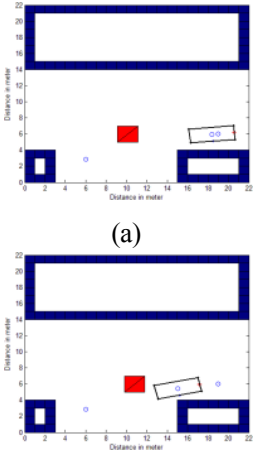

(d)

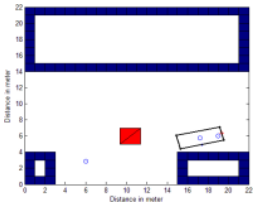

(b)

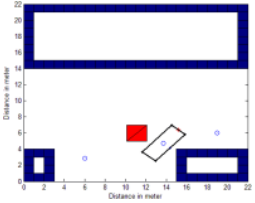

(e)

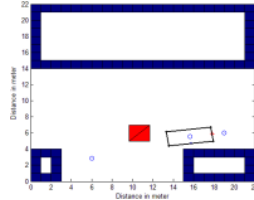

(c)

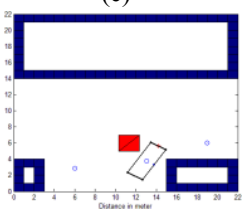

(f)

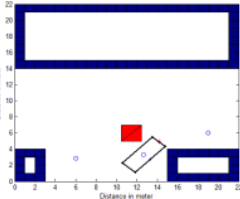

(g)

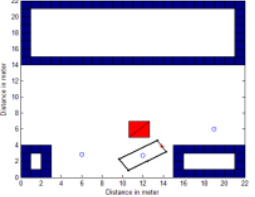

(h)

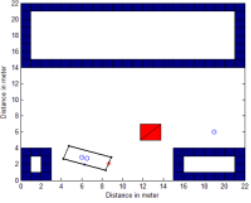

(i)
Fig. 11. Step sequence of reverse parallel parking with $\mathrm{K}=0.25$

\section{Conclusion}

In this paper, a fuzzy-based adaptive dimension parking algorithm integrated with an autonomous parking controller is developed. The obstacle avoidance system takes information from the ultrasonic sensor array that is assumed to mount on the peripheral of CLMR. This sensor information is grouped and fuzzified using the proposed algorithm based on vehicle dimensions. As shown from the simulation value of constant $\mathrm{K}$ can optimized path taken by CLMR. The value of $\mathrm{K}$ can be set heuristically or practically. This algorithm can be tested further to make it practically useable in commercial vehicles.

\section{References:}

[1] D. Lyon, "Parallel parking with curvature and nonholonomic constraints," IEEE Intell. Veh. Symp. Proc., pp. 341-346, 1992, doi: 10.1109/IVS.1992.252283.

[2] R. M. Murray and S. S. Sastry, "Nonholonomic motion planning. Steering using sinusoids," IEEE Trans. Automat. Contr., vol. 38, no. 5, pp. 700-716, 1993, doi: 10.1109/9.277235.

[3] J. P. Laumond, P. E. Jacobs, M. Taïx, and R. M. Murray, "A Motion Planner for Nonholonomic Mobile Robots," IEEE Trans. Robot. Autom., vol. 10, no. 5, pp. 577-593, 1994, doi: 10.1109/70.326564.

[4] I. E. Paromtchik and C. Laugier, "Motion generation and control for parking an autonomous vehicle," Proc. - IEEE Int. Conf. Robot. Autom., vol. 4, no. April, pp. $3117-$ 3122, 1996, doi: 10.1109/robot.1996.509186.

[5] S. J. Chang and T. H. S. Li, "Design and implementation of fuzzy parallel-parking control for a car-type mobile robot," J. Intell. Robot. Syst. Theory Appl., vol. 34, no. 2, pp. 175-194, 2002, 10.1023/A:1015664327686.

[6] T.-H. S. Li and Shih-Jie Chang, 
"Autonomous fuzzy parking control of a carlike mobile robot," IEEE Trans. Syst. Man, Cybern. - Part A Syst. Humans, vol. 33, no. 4, pp. 451-465, Jul. 2003, doi: 10.1109/TSMCA.2003.811766.

[7] T. H. S. Li, S. J. Chang, and Y. X. Chen, "Implementation of human-like driving skills by autonomous fuzzy behavior control on an FPGA-based car-like mobile robot," IEEE Trans. Ind. Electron., vol. 50, no. 5, pp. 867880, 2003, doi: 10.1109/TIE.2003.817490.

[8] I. Baturone, F. J. Moreno-Velo, S. SánchezSolano, and A. Ollero, "Automatic design of fuzzy controllers for car-like autonomous robots," IEEE Trans. Fuzzy Syst., vol. 12, no. 4, pp. 447-465, 2004, doi: 10.1109/TFUZZ.2004.832532.

[9] Y. Zhao and E. G. Collins, "Robust automatic parallel parking in tight spaces via fuzzy logic," Rob. Auton. Syst., vol. 51, no. $2-3$, pp. 111-127, 2005, doi: 10.1016/j.robot.2005.01.002.

[10] C. H. Chao, C. H. Ho, S. H. Lin, and T. H. S. $\mathrm{Li}$, "Omni-directional vision-based parallelparking control design for car-like mobile robot," Proc. 2005 IEEE Int. Conf. Mechatronics, ICM '05, vol. 2005, pp. 562567, 2005, doi: 10.1109/ICMECH.2005.1529319.

[11] G. Antonelli, S. Chiaverini, and G. Fusco, "A fuzzy-logic-based approach for mobile robot path tracking," IEEE Trans. Fuzzy Syst., vol. 15, no. 2, pp. 211-221, 2007, doi: 10.1109/TFUZZ.2006.879998.

[12] B. Müller, J. Deutscher, and S. Grodde, "Continuous curvature trajectory design and feedforward control for parking a car," IEEE Trans. Control Syst. Technol., vol. 15, no. 3, pp. 541-553, 2007, doi: 10.1109/TCST.2006.890289.

[13] W. C. Wang and R. Chen, "A vision-based fuzzy logic controller for backing-up an autonomous vehicle," J. Intell. Fuzzy Syst., vol. 19, no. 4-5, pp. 273-284, 2008.

[14] C.-K. Lee, C.-L. Lin, and B.-M. Shiu, "Autonomous Vehicle Parking Using Hybrid Artificial Intelligent Approach," J. Intell. Robot. Syst., vol. 56, no. 3, pp. 319-343,
Oct. 2009, doi: 10.1007/s10846-009-9319-9.

[15] K. Demirli and M. Khoshnejad, "Autonomous parallel parking of a car-like mobile robot by a neuro-fuzzy sensor-based controller," Fuzzy Sets Syst., vol. 160, no. 19, pp. 2876-2891, 2009, doi: 10.1016/j.fss.2009.01.019.

[16] T. H. S. Li, Y. C. Yeh, J. Da Wu, M. Y. Hsiao, and C. Y. Chen, "Multifunctional intelligent autonomous parking controllers for carlike mobile robots," IEEE Trans. Ind. Electron., vol. 57, no. 5, pp. 1687-1700, 2010, doi: 10.1109/TIE.2009.2033093.

[17] X. Du and K. K. Tan, "Autonomous reverse parking system based on robust path generation and improved sliding mode control," IEEE Trans. Intell. Transp. Syst., vol. 16, no. 3, pp. 1225-1237, 2015, doi: 10.1109/TITS.2014.2354423.

[18] H. Vorobieva, S. Glaser, N. Minoiu-Enache, and S. Mammar, "Automatic parallel parking in tiny spots: Path planning and control," IEEE Trans. Intell. Transp. Syst., vol. 16, no. 1, pp. 396-410, 2015, doi: 10.1109/TITS.2014.2335054.

[19] N. Nakrani and M. Joshi, "Fuzzy based Autonomous Parallel Parking Challenges in Real time Scenario," in Advances in Intelligent Systems and Computing, 2016, pp. $789-802$.

[20] G. Hongbo, X. Guotao, Z. Xinyu, and C. Bo, "Autonomous parking control for intelligent vehicles based on a novel algorithm," $J$. China Univ. Posts Telecommun., vol. 24, no. 4, pp. 51-56, 2017, doi: 10.1016/S10058885(17)60223-1.

[21] W. Liu, Z. Li, L. Li, and F. Y. Wang, "Parking Like a Human: A Direct Trajectory Planning Solution," IEEE Trans. Intell. Transp. Syst., vol. 18, no. 12, pp. 33883397, 2017, doi: 10.1109/TITS.2017.2687047.

[22] M. Ornik, M. Moarref, and M. E. Broucke, "An Automated Parallel Parking Strategy Using Reach Control Theory," IFACPapersOnLine, vol. 50, no. 1, pp. 90899094, 2017, doi: 10.1016/j.ifacol.2017.08.1699. 
[23] M. Heimberger, J. Horgan, C. Hughes, J. McDonald, and S. Yogamani, "Computer vision in automated parking systems: Design, implementation and challenges," Image Vis. Comput., vol. 68, pp. 88-101, 2017, doi: 10.1016/j.imavis.2017.07.002.

[24] N. Nakrani and M. Joshi, "An Intelligent Fuzzy based Hybrid Approach for Parallel Parking in Dynamic Environment," Procedia Comput. Sci., vol. 133, pp. 82-91, 2018, doi: 10.1016/j.procs.2018.07.011.

[25] D. Gorinevsky, A. Kapitanovsky, and A. Goldenberg, "Neural network architecture for trajectory generation and control of automated car parking," IEEE Trans. Control Syst. Technol., vol. 4, no. 1, pp. 50 56, 1996, doi: 10.1109/87.481766.

[26] W. L. Xu and S. K. Tso, "Sensor-based fuzzy reactive navigation of a mobile robot through local target switching," IEEE Trans. Syst. Man Cybern. Part C Appl. Rev., vol. 29, no. 3, pp. 451-459, 1999, doi: $10.1109 / 5326.777079$.

[27] M. M. Joshi and M. A. Zaveri, "Fuzzy based autonomous robot navigation system," Proc. INDICON 2009 - An IEEE India Counc. Conf., 2009, doi: 10.1109/INDCON.2009.5409419.

[28] C. L. Hwang and C. Y. Shih, "A distributed active-vision network-space approach for the navigation of a car-like wheeled robot," IEEE Trans. Ind. Electron., vol. 56, no. 3, pp. $\quad 846-855, \quad 2009, \quad$ doi: 10.1109/TIE.2008.2004388.

[29] M. M. Joshi and M. A. Zaveri, "Neuro-fuzzy based autonomous mobile robot navigation system," 11th Int. Conf. Control. Autom. Robot. Vision, ICARCV 2010, no. December, pp. 384-389, 2010, doi: 10.1109/ICARCV.2010.5707354.

[30] P. Krammer and H. Schweinzer, "Localization of object edges in arbitrary spatial positions based on ultrasonic data," IEEE Sens. J., vol. 6, no. 1, pp. 203-210, 2006, doi: 10.1109/JSEN.2005.860359.

[31] N. Petrellis, N. Konofaos, and G. P. Alexiou, "Target Localization Utilizing the Success Rate in Infrared Pattern Recognition," IEEE
Sens. J., vol. 6, no. 5, pp. 1355-1364, Oct. 2006, doi: 10.1109/JSEN.2006.881342.

[32] J. Reijniers and H. Peremans, "Biomimetic Sonar System Performing Spectrum-Based Localization," IEEE Trans. Robot., vol. 23, no. 6, pp. 1151-1159, Dec. 2007, doi: 10.1109/TRO.2007.907487.

[33] D. Bank and T. Kampke, "High-Resolution Ultrasonic Environment Imaging," IEEE Trans. Robot., vol. 23, no. 2, pp. 370-381, Apr. 2007, doi: 10.1109/TRO.2007.895060.

[34] D. A. Thornton, K. Redmill, and B. Coifman, "Automated parking surveys from a LIDAR equipped vehicle," Transp. Res. Part C Emerg. Technol., vol. 39, no. 2014, pp. 23-35, 2014, doi: 10.1016/j.trc.2013.11.014.

[35] J. K. Suhr and H. G. Jung, "Automatic Parking Space Detection and Tracking for Underground and Indoor Environments," IEEE Trans. Ind. Electron., vol. 63, no. 9, pp. 5687-5698, 2016, doi: 10.1109/TIE.2016.2558480.

\section{Creative Commons Attribution License 4.0 (Attribution 4.0 International, CC BY 4.0)}

This article is published under the terms of the Creative Commons Attribution License 4.0 https://creativecommons.org/licenses/by/4.0/deed.en US 